\title{
DESEMPENHO DE NOVILHAS DE CORTE ALIMENTADAS COM CANA HIDROLISADA
}

\author{
Felipe Nogueira Domingues ${ }^{1}$, Mauro Dal Secco Oliveira ${ }^{2}$, Diego AZevedo Mota ${ }^{3}$, Daniel De \\ SOUZA FERREIRA ${ }^{4}$, JULIANA SANTOS ${ }^{5}$ \\ ${ }^{1}$ Universidade Federal do Pará, Belém PA, Brasil - felipend@ ufpa.br \\ ${ }^{2}$ Professor da Faculdade de Agronomia e Veterinária da Universidade Estadual Paulista, Jaboticabal, SP, Brasil \\ ${ }^{3}$ Professor Adjunto da Universidade Federal do Amazonas (UFAM), Campus do Baixo Amazonas, Parintins, AM, Brasil \\ ${ }^{4}$ Colégio Técnico Agrícola da Universidade Estadual Paulista, Jaboticabal, SP, Brasil \\ ${ }^{5}$ Instituto Federal do Rio Grande do Sul/Campus de Sertão, Sertão, RS, Brasil
}

\begin{abstract}
Objetivou-se com este estudo avaliar o desempenho, o consumo e a conversão alimentar de novilhas Angus x Nelore alimentadas com cana-de-açúcar hidrolisada. Foram utilizadas 24 novilhas (1/2 sangue Angus x Nelore) com peso corporal médio de $242 \mathrm{~kg} \pm 23 \mathrm{~kg}$, confinadas em baias individuais com área total de $15 \mathrm{~m}^{2}$. O trabalho foi composto por seis tratamentos distribuídos da seguinte forma: T1 - cana in natura: a cana-de-açúcar era fornecida aos animais após picada; T2 - cana hidrolisada com $0,5 \%$ cal virgem e 24 horas de exposição ao ar; T3 cana hidrolisada com $0,5 \%$ cal virgem e 48 horas de exposição ao ar; T4 - cana hidrolisada com 1,0\% cal
\end{abstract}

virgem e 24 horas de exposição ao ar; T5 - cana hidrolisada com $1,0 \%$ cal virgem e 48 horas de exposição ao ar; T6 - cana hidrolisada com 1,0\% cal virgem e 72 horas de exposição ao ar. Utilizou-se o delineamento inteiramente casualizado com quatro repetições por tratamento, sendo as médias comparadas pelo teste de Tukey a $5 \%$ de significância de probabilidade. Não foram verificadas diferenças estatísticas em nenhum dos tratamentos $(\mathrm{P}>0,05)$. O tratamento da cana-de-açúcar com cal virgem não aumentou o consumo nem $o$ desempenho dos animais avaliados neste trabalho.

PALAVRAS-CHAVE: hidrólise; nutrição de ruminantes; óxido de cálcio; tratamento alcalino.

\section{PERFORMANCE OF BEEF HEIFERS FED HYDROLYSED SUGARCANE}

\section{ABSTRACT}

The objective of this study was to evaluate the performance, consumption and feed conversion of Angus $x$ Nellore heifers fed hydrolyzed sugarcane. Twenty-four (1/2 Angus x Nellore crossbred) heifers were used with average body weight of $242 \mathrm{~kg} \pm 23 \mathrm{~kg}$, confined in individual pens with total area of $15 \mathrm{~m}^{2}$. The study consisted of six treatments distributed as follows: T1 - in natura sugarcane: sugarcane was given to animals after being chopped; T2 - hydrolyzed sugarcane with $0.5 \%$ lime and 24 hours of air exposure; T3 - hydrolyzed sugarcane with $0.5 \%$ lime and 48 hours of air exposure;
T4 - hydrolyzed sugarcane with $1.0 \%$ lime and 24 hours of air exposure; T5 - hydrolyzed sugarcane with $1.0 \%$ lime and 48 hours of air exposure; T6 - hydrolyzed sugarcane with $1.0 \%$ lime and 72 hours of air exposure. We used a completely randomized design with four replicates per treatment, and averages were compared by Tukey test at $5 \%$ significant level of probability. No statistical difference was found for any of the treatments $(\mathrm{P}>0.05)$. The processing of sugarcane with lime did not increase the intake and performance of animals evaluated in this study.

KEYWORDS: alkaline treatment; calcium oxide; hydrolysis; nutrition ruminants. 


\section{INTRODUÇÃO}

A bovinocultura brasileira tem grande importância para o desenvolvimento nacional, visto que, além de ter o papel social de fixar o homem no meio rural, o leite, a carne e seus derivados têm presença marcante no hábito alimentar do brasileiro. Junto a isso, o Brasil nos últimos anos tem elevado potencialmente a sua participação no mercado internacional desses produtos.

Características como colheita na entressafra, alta produção de matéria seca por hectare, custo da matéria seca relativamente baixo, fácil manejo e pouco risco agronômico em comparação com as culturas tradicionalmente utilizadas como o milho para a produção de silagem, tem feito da cana-de-açúcar uma opção para a alimentação animal nas pequenas e grandes propriedades rurais. Essa forrageira inicialmente foi preconizada para ser utilizada na forma in natura e mais recentemente na forma de silagem.

No Brasil, um dos primeiros relatos da utilização da cana na alimentação animal foi feito na década de 40 (ATHANASSOF et al., 1940).

Dois pontos negativos são relevantes no estudo da cana-de-açúcar. O primeiro é em relação à baixa digestibilidade aparente da porção fibrosa da cana, que causa um baixo consumo de matéria seca, logo uma baixa ingestão de nutrientes. Assim, alternativas para aumentar a fração potencialmente digestível da fibra ou sua taxa de digestão podem ter impactos positivos sobre o consumo e o desempenho dos animais (PINA et al., 2011). O segundo está relacionado ao corte diário da cana que onera o custo de produção dos sistemas nos quais a cana está inserida e também reduz a qualidade de vida do produtor rural porque ele tem a necessidade de cortar a cana tanto nos finais de semana quanto nos feriados. Dessa forma, a estocagem da cana já picada por até 72 horas permitiria melhor logística para sua utilização e provável redução dos custos associados ao corte, transporte e desintegração.

Com o intuito de aumentar a digestibilidade da fração fibrosa dos mais diversos alimentos, a adição de alguns produtos químicos (agentes alcalinizantes hidrólise) sobre alguns ingredientes volumosos vem sendo estudada desde a década de 20. Dentre eles, os mais utilizados são o hidróxido de sódio, hidróxido de cálcio, amônia anidra e, mais recentemente, o óxido de cálcio. Esses agentes atuam solubilizando parcialmente a hemicelulose e promovem o fenômeno conhecido como "intumescimento alcalino da celulose", que consiste na expansão das moléculas de celulose, causando a ruptura das ligações das pontes de hidrogênio, as quais, segundo JACKSON (1977), conferem a cristalinidade da celulose, aumentando a digestão desta e da hemicelulose. De acordo com KLOPFENSTEIN (1978), o teor de lignina normalmente não é alterado pelo tratamento químico, mas a ação deste leva ao aumento da taxa de digestão da fibra.

Esses agentes alcalinizantes também seriam capazes de conservar o material já picado, isto é, reduzir a necessidade de cortes diários do canavial, podendo ocasionar uma melhora na logística de utilização da cana-de-açúcar.

Recentemente, artigos avaliando o desempenho de bovinos alimentados com cana hidrolisada (PINA et al., 2011; TEIXEIRA JÚNIOR, 2008; SFORCINI, 2009 e MORAES et al., 2008a) têm sido publicados; entretanto, os resultados são contraditórios, indicando a necessidade de maiores estudos avaliando a cana-de-açúcar hidrolisada na alimentação de ruminantes.

Objetivou-se com este estudo avaliar o desempenho, consumo e conversão alimentar de novilhas mestiças (Angus x Nelore) alimentadas com cana-de-açúcar hidrolisada.

\section{MATERIAL E MÉTODOS}

$\mathrm{O}$ experimento foi conduzido na Faculdade de Ciências Agrárias e Veterinárias da UNESP, Campus de Jaboticabal/SP, situada a $21^{\circ} 15^{\prime} 22^{\prime}$ " de latitude sul e $48^{\circ} 18^{\prime} 22^{\prime}$ ' de longitude oeste, a $610 \mathrm{~m}$ de altitude, com temperatura média anual de $22^{\circ} \mathrm{C}$, pluviosidade $1400 \mathrm{~mm} / \mathrm{ano}$ e umidade relativa do ar média de 70,8 $\%$.

A cana-de-açúcar utilizada foi o cultivar IAC 86-2480, plantada no campus da UNESP/Jaboticabal, sendo o corte realizado a partir do momento em que a mesma estava com 9 meses de idade ( $1^{\circ}$ corte). A canade-açúcar foi cortada com o auxílio de uma forrageira da marca Menta Mint modelo Premium Doblo ${ }^{\circledR}$ acoplada ao trator, sendo a máquina regulada para obtenção de partículas de 8 a $10 \mathrm{~mm}$.

A fase de campo do experimento foi realizada entre os meses de agosto e novembro de 2007. A duração total foi de 105 dias, sendo os 21 dias iniciais de adaptação dos animais à instalação e manejo e 84 dias de avaliação divididos em três períodos de 28 dias. Os animais foram pesados ao final de cada período após jejum hídrico e alimentar de 15 horas.

Foram utilizadas 24 novilhas (1/2 sangue Angus x Nelore) com peso corporal médio de $242 \mathrm{~kg} \pm$ $23 \mathrm{~kg}$, confinadas em baias individuais providas de cochos e comedouros com área total de $15 \mathrm{~m}^{2}$. Todos os animais foram identificados e submetidos ao controle de endo- e ectoparasitas.

Os animais foram distribuídos nos seguintes tratamentos:

T1 - cana in natura: a cana-de-açúcar era fornecida aos animais após picada.

$\mathrm{T} 2$ - cana hidrolisada com $0,5 \%$ cal virgem e 24 horas de exposição ao ar.

T3 - cana hidrolisada com $0,5 \%$ cal virgem e 48 horas 
de exposição ao ar.

T4 - cana hidrolisada com 1,0\% cal virgem e 24 horas de exposição ao ar.

T5 - cana hidrolisada com 1,0\% cal virgem e 48 horas de exposição ao ar.

T6 - cana hidrolisada com 1,0\% cal virgem e 72 horas de exposição ao ar.

As dietas experimentais foram compostas pela relação volumoso:concentrado 60:40 com base na matéria seca, com o objetivo de maximizar o efeito da cana-de-açúcar. Todos os animais receberam o mesmo concentrado, conforme apresentado na Tabela 1.

Tabela 1: Composição do concentrado (\%MN)

\begin{tabular}{|c|c|}
\hline Ingredientes & $\% \mathrm{MN}^{2}$ \\
\hline Milho & 48,38 \\
\hline Farelo de Soja & 43,12 \\
\hline Uréia & 2,5 \\
\hline Núcleo mineral ${ }^{1}$ & 6,0 \\
\hline $\begin{array}{l}\text { Composição (Níveis de } \\
40 \mathrm{~g} ; \mathrm{Ca}-146 \mathrm{~g} ; \mathrm{Na}-56 \\
-1300 \mathrm{mg} ; \mathrm{Mn}-900 \mathrm{~m} \\
\text { mg; Se - } 10 \mathrm{mg} ; \mathrm{F} \text { (max.) } \\
\text { 2Matéria Natural }\end{array}$ & $\begin{array}{l}\text { o com o fabricante): } \mathrm{P}- \\
-20 \mathrm{~g} ; \mathrm{Cu}-350 \mathrm{mg} ; \mathrm{Zn} \\
\mathrm{mg} ; \mathrm{Co}-10 \mathrm{mg} ; \mathrm{I}-24 \\
\text { t.s.q } 1000 \mathrm{~g} \text {. }\end{array}$ \\
\hline
\end{tabular}

De acordo com o fabricante, a cal virgem utilizada na hidrólise da cana-de-açúcar apresentou a seguinte composição química, $90 \%$ de $\mathrm{CaO}$ e $0,5 \%$ de $\mathrm{MgO}$.

As dietas foram calculadas de acordo com as exigências nutricionais apresentadas pelo NRC (1996), objetivando ganho médio diário de $1 \mathrm{~kg}$.

A alimentação foi fornecida, à vontade, em duas refeições diárias com quantidades iguais sendo a primeira às 6 horas e a segunda às 13 horas, visando à obtenção de sobras de 5 a $10 \%$ do fornecido.

Amostras do concentrado, volumoso e sobras de cada animal foram coletadas três vezes por semana. Essas amostras foram armazenadas em congelador a $-5^{\circ} \mathrm{C}$. Posteriormente, foram feitas amostras compostas por animal por período (28 dias) com base no peso seco.

As amostras compostas foram levadas ao Laboratório de Nutrição Animal da FCAV/Unesp para análise de matéria seca (MS), proteína bruta (PB), extrato etéreo (EE), matéria mineral (MM), matéria orgânica (MO) e fibra em detergente neutro ( FDN), segundo SILVA \& QUEIROZ (2002).

Todos os tratamentos que continham a cana hidrolisada foram feitos misturando-se a respectiva dose de cal (\%MN da cana-de-açúcar) de cada tratamento a quatro litros de água para cada $100 \mathrm{~kg}$ de cana-de-açúcar.

A cana-de-açúcar, depois de picada, foi espalhada em camadas de $\pm 20 \mathrm{~cm}$ sobre piso de concreto em galpão coberto sem paredes laterais. Em seguida, a calda contendo a respectiva dose de cal de cada tratamento foi distribuída sobre a cana, que foi homogeneizada até que o volumoso apresentasse a coloração amarela característica da cana-de-açúcar hidrolisada. Após a mistura, a cana foi amontoada, respeitando-se os tempos de exposição ao ar de cada tratamento para fornecimento aos animais.

A composição bromatológica do concentrado da cana-de-açúcar in natura e hidrolisada está descrita na Tabela 2.

A composição bromatológica das dietas totais (volumoso mais concentrado) encontra-se na Tabela 3.

Tabela 2: Composição bromatológica do concentrado e dos volumosos (cana-de-açúcar in natura e hidrolisada) utilizados nas dietas experimentais, em diferentes tempos de exposição ao ar

\begin{tabular}{lccccccc}
\hline \multirow{2}{*}{ Item } & \multirow{2}{*}{ Concentrado } & \multicolumn{7}{c}{ Volumoso (cana-de-açúcar) } \\
\cline { 2 - 7 } & & $\mathrm{T} 1$ & $\mathrm{~T} 2$ & $\mathrm{~T} 3$ & $\mathrm{~T} 4$ & $\mathrm{~T} 5$ & $\mathrm{~T} 6$ \\
\hline $\mathrm{MS}$ & 89,16 & 29,18 & 30,97 & 28,83 & 31,47 & 30,15 & 31,60 \\
$\mathrm{~PB}^{1}$ & 34,17 & 1,95 & 2,05 & 2,50 & 2,00 & 2,27 & 2,01 \\
$\mathrm{MM}^{1}$ & 10,15 & 4,92 & 7,37 & 7,88 & 9,01 & 10,18 & 9,84 \\
$\mathrm{MO}^{1}$ & 89,85 & 95,08 & 92,63 & 92,12 & 90,99 & 89,82 & 90,16 \\
$\mathrm{FDN}^{1}$ & 16,37 & 49,03 & 62,36 & 65,35 & 61,62 & 63,21 & 62,67 \\
$\mathrm{EE}^{1}$ & 3,83 & 0,81 & 0,96 & 1,07 & 0,78 & 1,28 & 0,66 \\
$\mathrm{Ca}^{1,2,3}$ & 1,09 & 0,20 & 1,23 & 1,28 & 2,11 & 2,26 & 2,30 \\
$\mathrm{P}^{1,2,3}$ & 0,58 & 0,12 & 0,12 & 0,11 & 0,12 & 0,12 & 0,11 \\
$\mathrm{Ca}: \mathrm{P}$ & $1,82: 1$ & $1,67: 1$ & $10,25: 1$ & $11,64: 1$ & $17,58: 1$ & $18,83: 1$ & $20,91: 1$ \\
\hline
\end{tabular}

${ }^{2}$ Os teores de Ca e P do concentrado são os valores descritos por VALADARES FILHO et al. (2002) para o grão de milho moído, farelo de soja e uréia e os valores descritos pelo fabricante do suplemento mineral.

${ }^{3}$ Os valores de Ca e P do volumoso apresentados nesta tabela são baseados nos resultados obtidos no trabalho de DOMINGUES (2009).

$\mathrm{T} 1$ - cana in natura. $\mathrm{T} 2$ - cana hidrolisada com $0,5 \% \mathrm{CaO}$ e 24 horas de exposição ao ar. T3 - cana hidrolisada com $0,5 \% \mathrm{CaO}$ e 48 horas de exposição ao ar. T4 - cana hidrolisada com 1,0\% $\mathrm{CaO}$ e 24 horas de exposição ao ar. T5 - cana hidrolisada com $1,0 \% \mathrm{CaO}$ e 48 horas de exposição ao ar. T6 - cana hidrolisada com 1,0\% CaO e 72 horas de exposição ao ar. 
Tabela 3: Composição bromatológica e teores de Ca e P das dietas totais (volumoso mais concentrado)

\begin{tabular}{lcccccc}
\hline \multirow{2}{*}{ Item } & \multicolumn{7}{c}{ Dietas experimentais ${ }^{2}$} \\
\cline { 2 - 6 } & $\mathrm{T} 1$ & $\mathrm{~T} 2$ & $\mathrm{~T} 3$ & $\mathrm{~T} 4$ & $\mathrm{~T} 5$ & T6 \\
\hline $\mathrm{MS}$ & 39,47 & 40,85 & 39,07 & 41,27 & 40,17 & 41,37 \\
$\mathrm{~PB}^{1}$ & 14,34 & 13,95 & 14,77 & 13,80 & 14,29 & 13,78 \\
$\mathrm{MM}^{1}$ & 6,93 & 8,40 & 8,76 & 9,43 & 10,17 & 9,95 \\
$\mathrm{MO}^{1}$ & 93,07 & 91,60 & 91,24 & 90,27 & 89,83 & 90,05 \\
$\mathrm{FDN}^{1}$ & 36,47 & 45,32 & 46,37 & 45,02 & 45,56 & 45,73 \\
$\mathrm{EE}^{1}$ & 1,97 & 2,02 & 2,14 & 1,90 & 2,24 & 1,82 \\
$\mathrm{Ca}^{1}$ & 0,56 & 1,17 & 1,20 & 1,70 & 1,79 & 1,82 \\
$\mathrm{P}^{1}$ & 0,30 & 0,30 & 0,30 & 0,30 & 0,30 & 0,30 \\
$\mathrm{Ca}: \mathrm{P}$ & $1,87: 1$ & $3,9: 1$ & $4,0: 1$ & $5,67: 1$ & $5,97: 1$ & $6,07: 1$ \\
\hline
\end{tabular}

${ }^{1} \% \mathrm{MS}$.

${ }^{2}$ Dieta total com relação volumoso:concentrado de 60:40.

$\mathrm{T} 1$ - cana in natura. T2 - cana hidrolisada com $0,5 \% \mathrm{CaO}$ e 24 horas de exposição ao ar. T3 - cana hidrolisada com $0,5 \% \mathrm{CaO}$ e 48 horas de exposição ao ar. T4 - cana hidrolisada com 1,0\% CaO e 24 horas de exposição ao ar. T5 - cana hidrolisada com 1,0\% CaO e 48 horas de exposição ao ar. T6 - cana hidrolisada com 1,0\% CaO e 72 horas de exposição ao ar.

Utilizou-se o delineamento inteiramente casualizado com 4 repetições por tratamento, sendo as médias comparadas pelo teste de Tukey em nível de significância de $5 \%$ de probabilidade.

\section{RESULTADOS E DISCUSSÃO}

A cana-de-açúcar utilizada neste estudo foi o cultivar IAC 86-2480 que, de acordo com LANDELL et al. (1997 e 2002), é uma variedade de maturação precoce. MARGARIDO (2006) comentou que cultivares de cana-de-açúcar considerados precoces podem ser utilizados a partir do mês de julho do ano subsequente ao plantio, independentemente se a cana foi plantada de setembro a novembro (cana-de-ano) ou plantadas de janeiro a abril (cana de ano e meio) (SEGATO et al., 2006). Dessa forma, apesar de a cana-de-açúcar estar apenas com nove meses de crescimento, a mesma apresentava condição satisfatória para o fornecimento aos animais.

Segundo BERGER et al. (1994), o objetivo do tratamento de forragens é aumentar o consumo de alimentos com alto teor de fibra, com base na melhora na taxa ou extensão de digestão e na disponibilidade de nutrientes.

A partir dos resultados apresentados na Tabela 4, verifica-se que não houve efeito significativo em nenhuma variável, com exceção do consumo de FDN em \%PC, que foi superior nos animais que receberam a dieta do $\mathrm{T} 4$ em relação aos animais que receberam a dieta do $\mathrm{T} 1$.

Tabela 4: Consumo de nutrientes em novilhas alimentadas com cana-de-açúcar hidrolisada com diferentes doses de cal e diferentes tempos de exposição ao ar

\begin{tabular}{|c|c|c|c|c|c|c|c|c|}
\hline \multirow{2}{*}{ Item } & $\mathrm{T} 1$ & $\mathrm{~T} 2$ & $\mathrm{~T} 3$ & $\mathrm{~T} 4$ & T5 & T6 & \multirow{2}{*}{ Média } & \multirow{2}{*}{$\mathrm{CV}(\%)$} \\
\hline & \multicolumn{6}{|c|}{$\mathrm{Kg} / \mathrm{dia}^{1}$} & & \\
\hline MS & 5,83 & 6,29 & 5,82 & 6,62 & 5,12 & 6,01 & 5,95 & 22,63 \\
\hline MO & 5,41 & 5,75 & 5,29 & 5,99 & 4,60 & 5,40 & 5,41 & 22,84 \\
\hline MM & 0,42 & 0,53 & 0,52 & 0,63 & 0,53 & 0,60 & 0,54 & 20,96 \\
\hline PB & 0,88 & 0,92 & 0,93 & 0,96 & 0,79 & 0,90 & 0,90 & 22,55 \\
\hline FDN & 1,91 & 2,68 & 2,50 & 2,85 & 2,20 & 2,57 & 2,45 & 22,15 \\
\hline EE & 0,12 & 0,14 & 0,14 & 0,13 & 0,13 & 0,12 & 0,13 & 20,42 \\
\hline \multicolumn{9}{|c|}{$\% \mathrm{PC}^{2}$} \\
\hline MS & 2,02 & 2,21 & 2,11 & 2,35 & 1,89 & 2,20 & 2,13 & 15,01 \\
\hline MO & 1,87 & 2,02 & 1,92 & 2,13 & 1,69 & 1,98 & 1,93 & 15,14 \\
\hline FDN & $0,66 \mathrm{~b}$ & $0,94 \mathrm{ab}$ & $0,91 \mathrm{ab}$ & $1,00 \mathrm{a}$ & $0,81 \mathrm{ab}$ & $0,94 \mathrm{ab}$ & 0,88 & 15,22 \\
\hline
\end{tabular}

Médias nas linhas seguidas da mesma letra minúscula não diferem estatisticamente pelo teste de Tukey $(\mathrm{P}>0,05)$.

${ }^{1}$ Consumo de nutrientes em $\mathrm{kg} / \mathrm{dia}$

${ }^{2}$ Consumo de nutrientes em $\%$ peso corporal

$\mathrm{T} 1$ - cana in natura. T2 - cana hidrolisada com $0,5 \% \mathrm{CaO}$ e 24 horas de exposição ao ar. T3 - cana hidrolisada com $0,5 \% \mathrm{CaO}$ e 48 horas de exposição ao ar. T4 - cana hidrolisada com 1,0\% CaO e 24 horas de exposição ao ar. T5 - cana hidrolisada com 1,0\% CaO e 48 horas de exposição ao ar. T6 - cana hidrolisada com 1,0\% CaO e 72 horas de exposição ao ar. 
A semelhança nos valores de consumo sugere que a atuação da cal sobre a fibra (hidrólise) não foi suficiente para estimular o consumo da cana hidrolisada. Esses resultados estão de acordo com PINA et al. (2011) que, ao avaliarem o desempenho de novilhas Nelores alimentadas com cana hidrolisada com três doses de cal $(0 ; 0,5 ;$ e $1,0 \%$ em relação a MN da cana) e dois tempos de exposição ao ar (0 e 3 dias), não observaram alterações no consumo de MS, MO, PB e NDT. Nesse mesmo estudo, os autores observaram aumento no consumo dos componentes da parede celular similar ao presente trabalho, no qual apenas o consumo de FDN em relação ao peso corporal sofreu acréscimo em relação à cana in natura.

O aumento no consumo de FDN foi resultado do aumento na concentração de FDN nas dietas que continham a cana hidrolisada (Tabela 3); no entanto, esse aumento no consumo de fibra, provavelmente, não está relacionado ao conhecido poder de hidrólise da cal virgem. O que de fato ocorreu é que todos os tratamentos que continham a cana hidrolisada ficaram armazenados entre 24 e 72 horas e, durante a estocagem, ocorre $\mathrm{o}$ desenvolvimento de microrganismos deterioradores que consomem os carboidratos solúveis da cana, causando o aumento percentual de FDN na cana hidrolisada e, consequentemente, aumentando a sua ingestão. Tal crescimento de microrganismos foi descrito por DOMINGUES et al. (2011).

Apesar da semelhança estatística dos resultados de consumo, alguns parâmetros de consumos devem ser observados de maneira criteriosa.

Os animais que receberam a dieta do $\mathrm{T} 4$ foram os que apresentaram os maiores valores numéricos para o consumo dos nutrientes avaliados, e isso é explicado pelo maior consumo absoluto de MS.

Os valores numéricos de consumo de MM ( $\mathrm{kg} / \mathrm{dia})$ aumentaram com o tratamento da cana com a cal virgem. Esse resultado era esperado devido à elevação nos teores de MM das dietas que continham a cal.

De acordo com os resultados apresentados na Tabela 5, verifica-se que não houve efeito significativo para o GMD e CA alimentar dos animais.

Tabela 5: Ganho médio diário (GMD - kg/dia) e conversão alimentar (CA - kg MS/kg GMD) de novilhas alimentadas com cana-de-açúcar hidrolisada com diferentes doses de cal e diferentes tempos de exposição ao ar

\begin{tabular}{lcccccccc}
\hline Item & T1 & T2 & T3 & T4 & T5 & T6 & Média & CV $^{1}(\%)$ \\
\hline GMD & 1,13 & 1,09 & 0,98 & 1,00 & 0,91 & 0,87 & 1,00 & 25,35 \\
CA & 5,59 & 5,77 & 5,93 & 6,51 & 5,58 & 6,87 & 6,04 & 16,38 \\
\hline
\end{tabular}

Médias nas linhas seguidas da mesma letra minúscula não diferem estatisticamente pelo teste de Tukey $(\mathrm{P}>0,05)$.

${ }^{1}$ Coeficiente de variação

$\mathrm{T} 1$ - cana in natura. T2 - cana hidrolisada com $0,5 \% \mathrm{CaO}$ e 24 horas de exposição ao ar. T3 - cana hidrolisada com $0,5 \% \mathrm{CaO}$ e 48 horas de exposição ao ar. T4 - cana hidrolisada com 1,0\% CaO e 24 horas de exposição ao ar. T5 - cana hidrolisada com $1,0 \% \mathrm{CaO}$ e 48 horas de exposição ao ar. T6 - cana hidrolisada com 1,0\% CaO e 72 horas de exposição ao ar.

A igualdade dos resultados de desempenho nos tratamentos mostra que a cana hidrolisada permite ganhos médios diários semelhantes em períodos de estocagem de até 72 horas quando comparados à cana in natura; contudo, observandose os valores absolutos, verifica-se que os tratamentos constituídos com a cana hidrolisada foram numericamente inferiores, chegando à redução de $23,00 \%$ no desempenho dos animais em relação aos tratamentos T1 (T1 - cana in natura) e T6 (cana hidrolisada com $1,0 \% \mathrm{CaO}$ e 72 horas de exposição ao ar).

Um dos motivos que justifica a hidrólise da cana-de-açúcar seria o aumento no consumo de MS, logo, melhor desempenho dos animais; entretanto, estudos que avaliaram o desempenho de animais ruminantes são contraditórios como pode ser verificado em seguida.

TEIXEIRA JÚNIOR (2008) observou aumento de $1,3 \mathrm{~kg}$ de leite/vaca/dia com a cana hidrolisada com $0,5 \%$ de cal virgem com base na matéria natural da cana quando comparada à cana in natura, assim como notou menor custo do quilograma de leite produzido em relação à silagem de milho e à cana in natura picada. Convém ressaltar que mesmo não havendo aumento no consumo de matéria seca, pode ocorrer aumento na produção de leite, uma vez que a hidrólise atua na melhoria da digestibilidade da porção fibrosa da cana. Além disso, SFORCINI (2009) verificou aumento na produção de leite de vacas alimentadas com cana hidrolisada com cal quando comparada à cana in natura.

MORAES et al. (2008a) afirmam que a 
cana-de-açúcar com a adição de $1 \%$ de $\mathrm{CaO}$ fornecida após 24 horas de armazenamento prejudica o consumo e o desempenho dos animais. Segundo os autores, é provável que a diminuição no consumo de matéria seca da cana hidrolisada e, consequentemente, o pior desempenho, tenha sido devido ao fato de a aceitabilidade ser pior nas dietas com a cana hidrolisada.

FREITAS et al. (2008), no estudo com ovinos, comentaram que não foi observado benefício do tratamento da cana-de-açúcar com hidróxido de cálcio, na dose de $0,5 \%$, tendo ocorrido redução no ganho de peso para o tratamento com $0,9 \%$ de cal.

Segundo PINA et al. (2011), no estudo com novilhas Nelore, o tempo de exposição ao ar da canade-açúcar de até 72 horas não influenciou o desempenho dos animais; contudo, a dose de inclusão de cal reduziu linearmente o ganho de peso dos animais. Ainda segundo esses autores, a possível estocagem da cana-de-açúcar por um período de 72 horas seria umas das vantagens do processo de hidrólise, porque melhoraria a logística e reduziria os custos associados com corte, transporte e trituração do material.

É interessante ressaltar que os trabalhos de desempenho que apresentaram resultados favoráveis à utilização da cana-de-açúcar hidrolisada envolveram vacas em lactação (TEIXEIRA JÚNIOR, 2008; SFORCINI, 2009) e que, nos estudos nos quais foram utilizados animais de corte e foi avaliado o GMD, o resultado foi desfavorável à utilização da cana hidrolisada (MORAES et al. 2008; PINA et al., 2011). Essa diversidade de resultados sugere o efeito expressivo da categoria animal em estudo.

Cabe ressaltar que alguns estudos foram desenvolvidos para avaliar o efeito da hidrólise da cana-de-açúcar por meio da cal virgem sobre os parâmetros ruminais, metabolismo de compostos nitrogenados, digestibilidade e consumo de bovinos e, na grande maioria desses estudos, a hidrólise da cana-de-açúcar não se demonstrou como sendo uma técnica promissora, sendo muitas vezes não recomendada (MORAES et al. 2008b; CARVALHO et al., 2010; CARVALHO et al., 2011).

\section{CONCLUSÃO}

A hidrólise da cana-de-açúcar com cal virgem não aumentou o consumo e o desempenho dos animais avaliados neste trabalho.

\section{REFERÊNCIAS}

ATHANASSOF, N. Cana na alimentação dos animais domésticos. Revista de Agricultura, v.15, p. 421-427, 1940.

BERGER, L.L.; FAHEY JR. , G.C.; BOURQUIN, L.D.; TITGEMEYER, E.C. Modification of forage quality after harvest. In: FAHEY Jr., G.C., COLLINS, M.; MOSER, L.E. and MERTENS, D.R. (Eds.) Forage quality, evaluation and utilization. Madison: American Society of Agronomy, 1994. p. 922-966.

CARVALHO, G.G.P.; GARCIA, R.; PIRES, A.J.V.; DETMANN, E.; SILVA, R.R.; PEREIRA, M.L.A.; SANTOS, A.B.; PEREIRA, T.C.J. Metabolismo de nitrogênio em novilhas alimentadas com dietas contendo cana-de-açúcar tratada com óxido de cálcio. Revista Brasileira de Zootecnia, v. 40, n. 3, p. 622-629, 2011.

CARVALHO, G.G.P.; GARCIA, R.; PIRES, A.J.V.; SILVA, R.R.; SANTANA JÚNIOR, H.A.; PINHEIRO, A.A.; MENDES, F.B.L.; RIBEIRO, L.S.O. Consumo e digestibilidade aparente em novilhas alimentadas com dietas contendo cana-de-açúcar tratada com óxido de cálcio. Revista Brasileira de Zootecnia, v. 39, n. 12, p. 2703-2713, 2010.

DOMINGUES, F.N. Cana-de-açúcar hidrolisada com doses crescentes de cal virgem e tempos de exposição ao ar para a alimentação de bovinos. 2009. 93f. Tese (Doutorado) - FCAV/UNESP, Jaboticabal. Disponível em http://capesdw.capes.gov.br/capesdw/resumo.html?idtese= $20092533004102002 \mathrm{P} 0$

DOMINGUES, F.N.; OLIVEIRA, M.D.S.; SIQUEIRA, G.R.; ROTH, A.P.T.P.; SANTOS, J. MOTA, D.A. Estabilidade aeróbia, pH e dinâmica de desenvolvimento de microrganismos da cana-de-açúcar in natura hidrolisada com cal virgem. Revista Brasileira de Zootecnia, v. 40, n. 4, p. 715-719, 2011.

FREITAS, A.W.P.; ROCHA F.C.; ZONTA, A.; FAGUNDES, J.L.; FONSECA, R.; ZONTA, M.C.M.; MACEDO, F.L. Consumo de nutrientes e desempenho de ovinos alimentados com dietas à base de cana-de-açúcar hidrolisada. Pesquisa Agropecuária Brasileira, v. 43, p. 1569-1574, 2008.

JACKSON, M.G. The alkali treatments of straws. Animal Feed Science and Technology, v. 2, n. 2, p. 105-130, 1977.

KLOPFENSTEIN, T. Chenical treatment of crop residues. Journal of Animal Science, v. 46, n. 3, p. 841-848, 1978.

LANDELL, M.G.A., CAMPANA, M.P.; RODRIGUES, A.A.; CRUZ, G.M.; BATISTA, L.A.R.; FIGUEIREDO, P.; SILVA, M.A.; BIDÓIA, M.A.P.; ROSSETTO, R.; MARTINS, A.L.M.; GALLO, P.B.; KANTHACK, R.A.D.; CAVICHIOLI, J.C.; VASCONCELOS, A.C.M.; XAVIER, M.A. A variedade IAC 86-2480 como nova opção de cana-de-açúcar para fins forrageiros: manejo de produção de uso na alimentação animal. Campinas: Instituto Agronômico (Boletim técnico, 193), 2002. 36p.

LANDELL, M.G.A.; CAMPANA, M.P.; FIGUEIREDO, P.; ZIMBACK, L.; SILVA, M.A.; PRADO, H. Novas variedades de cana-de-açúcar. Campinas: Instituto 
Agronômico (Boletim técnico, 169), 1997.

MARGARIDO, F.B. Planejamento agrícola em cana-deaçúcar. In: SEGATO, S.V.; PINTO, A.S.; JENDIROBA, E.; NOBREGA, J.C.M. (Eds), Atualização em produção de cana-de-açúcar. Livroceres, Piracicaba, 2006. p. 6978 .

MORAES, K.A.K.; VALADARES FILHO, S.C.; MORAES, E.H.B.K.; LEÃO, M.I.; VALADARES, R.F.D.; PEREIRA, O.G.; SÓLERO, B.P. Cana-de-açúcar tratada com óxido de cálcio fornecida com diferentes níveis de concentrado para novilhas de corte em confinamento. Revista Brasileira de Zootecnia, v. 37, n. 7, p. 1293-1300, 2008a.

MORAES, K.A.K.; VALADARES FILHO, S.C.; MORAES, E.H.B.K.; LEÃO, M.I.; VALADARES, R.F.D.; DETMANN, E.; NALON, P.M. Parâmetros ruminais de novilhas de corte alimentadas com cana-deaçúcar tratada com óxido de cálcio e diferentes níveis de concentrado. Revista Brasileira de Zootecnia, v. 37, n. 7, p. 1301-1310, 2008b.

NATIONAL RESEARCH COUNCIL. Nutrient requirements of beef cattle, $7^{\mathrm{a}} \mathrm{ed}$. Washington, National Academy Press. 1996. 242p.

PINA, D.S.; VALADARES FILHO, S.C.; TEDESCHI, L.O.; BARBOSA, A.M.; AZEVÊDO, J.A.G.; VALADARES, R.F.D.; SOUZA, N.K.P.; FONSECA, M.A. Níveis de inclusão e tempo de exposição da cana-de- açúcar ao óxido de cálcio sobre parâmetros digestivos e o desempenho de novilhas Nelore. Revista Brasileira de Zootecnia, v. 40, n. 3, p. 648-656, 2011.

SEGATO, S.V.; MATTIUZ, C.F.M.; MOZAMBANI, A.E. 2006. Aspectos fenológicos da cana-de-açúcar. In: SEGATO, S.V., PINTO, A.S.; JENDIROBA, E.; NOBREGA, J.C.M. (Eds), Atualização em produção de cana-de-açúcar, Piracicaba: Livroceres, 2006. p. 19-36.

SFORCINI, M.P.R. Silagem de milho e cana-de-açúcar in natura hidrolisada para vacas em lactação. Dissertação (Mestrado). Unesp/FCAV. Jaboticabal, SP. 93f, 2009. Disponível em: http://capesdw.capes.gov.br/capesdw/resumo.html?idtese= 20091133004102002P0

SILVA, D.J.; QUEIROZ, A.C. Análise de alimentos: métodos químicos e biológicos. 3.ed. Viçosa:UFV. 2002. 235p.

TEIXEIRA Jr, D.J. Hidrólise da cana-de-açúcar com cal virgem e cal hidratada na alimentação de vacas leiteiras. Dissertação (Mestrado) - FCAV/UNESP, Jaboticabal, SP. 46f, 2008. Disponível em: http://capesdw.capes.gov.br/capesdw/resumo.html?idtese= 20083033004102002P0

VALADARES FILHO, S. de C.; ROCHA JÚNIOR, V.R.; CAPPELLE, E.R. Tabelas brasileiras de composição de alimentos para bovinos CQBAL 2.0. Viçosa: UFV, 2002. 297p. 